Ausstellung an der Charité

\section{SICK! Kranksein im Comic}

\begin{abstract}
— Diagnosen wie Alzheimer, Depressionen oder Morbus Crohn verändern das Leben von Betroffenen, ihren Angehörigen und Freunden. Doch wie wird die Krankheit erlebt? Wie kann man lernen, damit umzugehen? Diesen Fragen widmen sich seit einigen Jahren verstärkt auch Comics. Eine Ausstellung im Berliner Medizinhistorischen Museum (BMM) der Charité beschäftigt sich mit Erzähl- und Darstellungs-
\end{abstract} weisen vom kurzen Comic-Strip bis zur Graphic Novel.

In der von der Kunsthistorikerin und Kulturwissenschaftlerin Dr. Uta Kornmeier kuratierten Ausstellung im medizinischen Präparatesaal des BMM werden Comics aus acht verschiedenen Ländern präsentiert, die persönlich, kritisch und mitunter auch humorvoll die vielfältigen Erfahrungen von Diagnose, Therapie, Behinderung sowie Pflege und Alltagserleben thematisieren. Die Ausstellungsform der Intervention ermöglicht einen Dialog zwischen Kunst und Wissenschaft: Die individuellen Krankengeschichten werden dabei in Bezug zu den anonymisierten medizinischen Präparaten Rudolf Virchows gesetzt. Die historischen Präparate, die auch heute noch für die Lehre genutzt werden, präsentieren

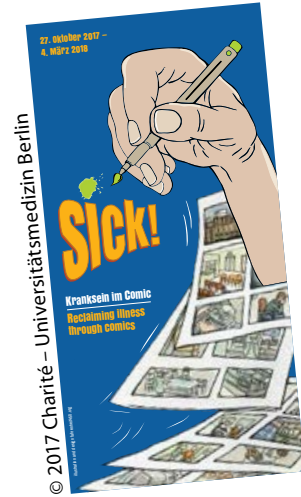

krankhafte Veränderungen des menschlichen Körpers in entpersonalisierter Form und aus Perspektive der Medizin. Ausgespart bleibt das emotionale und persönliche Erleben der Patienten, Angehörigen und medizinisch Tätigen. Diese kommen jetzt in den ausgestellten Comics zu Wort, um ihre Sichtweise auf Kranksein und Behinderung darzustellen.

Die Ausstellung „SICK! Kranksein im Comic / Reclaiming Illness through Comics" ist noch bis 4. März 2018 im Berliner Medizinhistorischen Museum zu sehen. Das Projekt ist an der Friedrich-Schlegel-Graduiertenschule für literaturwissenschaftliche Studien der Freien Universität Berlin angesiedelt und wird von der Einstein-Stiftung Berlin gefördert.

www.charite.de

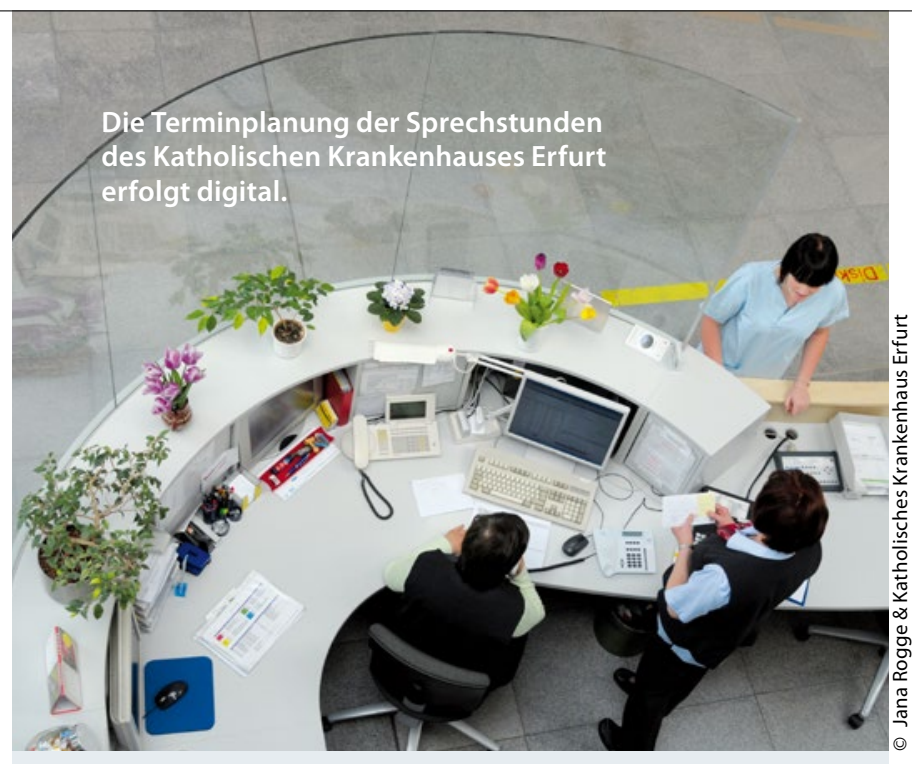

Krankenhaus Erfurt

\section{Terminplanung digital}

— Die Terminplanung im Katholischen Krankenhaus "St. Johann Nepomuk" in Erfurt läuft ab sofort online über den Ärzteservice Doctolib. Das Krankenhaus vereinfacht dadurch das Buchungsmanagement für Patienten und Mitarbeiter und geht einen weiteren Schritt in Richtung Digitalisierung.

Buchbar sind Sprechstunden der Klinik für Allgemein-, Visceral-, Thorax- und Gefäßchirurgie. Der Service soll sukzessive für alle ambulanten Sprechstunden ausgeweitet werden sowie für stationäre Untersuchungen und in der Zusammenarbeit mit niedergelassenen Ärzten zum Einsatz kommen. Patienten buchen ihre Termine online selbstständig, stornieren und organisieren sie. Dank automatischer Terminerinnerungen per SMS oder E-Mail erscheinen die Patienten zuverlässiger. Bei der Buchung können zudem Unterlagen wie Fragebögen vorab an den Patienten übermittelt werden. Der Service läuft in Echtzeit, so dass Patienten nur tatsächlich freie Termine buchen können.

„Die Wartezeiten für Patienten nehmen ab, die Mitarbeiter in den Sekretariaten, den Sprechstunden und der Anmeldung werden entlastet", so Dr. med. Martin Krajci. Als Leiter Medizinmanagement und Ambulanz ist er für die Einführung von digitalen Services verantwortlich.

www.kkh-erfurt.de

Für mehr Mobilität im Alter

\title{
Der Komfortlinienbus rollt an
}

— Iveco Bus, DB Regio Bus und die Hochschule Fresenius arbeiten gemeinsam an der Entwicklung eines neuen Linienbusmodells, mit dem künftig ältere oder in der Mobilität eingeschränkte Personen sicherer ihre Wegstrecken zurücklegen können. Aufgrund der Forschungsergebnisse der Hochschule Fresenius zur "Mobilität im Alter" wurden einige technische Veränderungen im Bus umgesetzt. So wird es künftig möglich sein, beispielsweise mit einem Rollator direkt beim Fahrer einzusteigen. Neben dem Sicherheitsaspekt spielt hier auch der psychologische Effekt, unmittelbar mit dem Fahrer in Kontakt zu kommen, eine große Rolle. Der Weg zu einem geeigneten Sitzplatz ist kürzer und wegen der breiteren Gänge bequemer. Die Sitze sind deutlich erhöht, was das Setzen und Aufstehen stark vereinfacht. Nicht zuletzt sind die Sitze alle in Fahrtrichtung angeordnet. Das wirkt sich positiv auf die Orientierung während der Fahrt aus.

Profitieren werden vor allem Menschen, die abseits der großen Städte leben. Für die Fahrten zum Arzt, zur Apotheke oder zum Einkaufen sind sie auf Mobilität angewiesen.

www.hs-fresenius.de 